\title{
Optimal Power System Control Using Artificial Neural Networks
}

\author{
V. ANIL \\ Assistant Professor \\ ANITS, Sangivalasa \\ M . Divakar \\ Assistant Professor \\ ANITS, Sangivalasa \\ L Dinesh \\ Assistant Professor \\ ANITS, Sangivalasa
}

\begin{abstract}
Electric power system is a highly complex and non linear system. Its analysis and control in real time environment requires highly sophisticated computational skills. Computations are reaching a limit as far as conventional computer based algorithms are concerned. It is therefore required to find out newer methods which can be easily implemented on dedicated hardware. It is a very difficult task due to complexity of the power system with all its interdependent variables, thus making the neural networks one of the better options for the solution of different issues in operation and control. In this project an attempt has been made to implement ANN's for observability determination, State Estimation, Economic Load Dispatch and for Reactive Power Optimization. A Hopfield neural network model has been developed to test Topological Observability of Power System and it is tested on two different test systems. The results so obtained, are comparable with those results of conventional root based observability determination technique. Further a Hopfield model has been developed to determine State Estimation of power system. State Estimation of 6 bus system and IEEE 14 bus system is attempted using this Hopfield neural network. Results obtained by developed model are compared with those of conventional Non Linear WLS State Estimation. Next use of ANN for Economic Load Dispatch problem has been developed. Economic Load dispatch has been studied using various test system data (like 3, 6, 20 \& 30 units) and the results are compared with conventional Lambda iterative technique and Particle Swarm Optimization techniques. Next Reactive Power Optimization problem has been attempted using ANN. The performance of so developed ANN is tested on Ward Hale 6 bus system and IEEE 30 bus system data and the results obtained are compared with those of the results obtained by GA and Particle Swarm Optimization technique.
\end{abstract}

Keywords: ANN, Load dispatch, optimization

DOI: $10.7176 / \mathrm{ISDE} / 10-6-01$

Publication date:July $31^{\text {st }} 2019$

\section{INTRODUCTION}

Modern Power System Control Centers must operate and control medium and large electrical networks that usually cover a large geographic area. The accomplishment of these tasks has been made possible due to important developments in computer and communication technologies. Now days, complex Power Systems require a very accurate and efficient control that makes Control Centers especially important. These centers are equipped with SCADA (Supervisory Control and Data Acquisition) systems allowing to acquire information about the Power System and its transmission to Control Centers in real-time. Due to the symbolic nature of the reasoning involved in some control tasks, Artificial Intelligence techniques appeared as a promising solution. Some years ago, some of the most important electrical utilities around the world began to develop knowledge-based applications to incorporate in their Control Centers. These applications deal with the information available in the Control Center and help operators in decision making. These methods must be capable of meeting all the important tasks of efficient control system such as numerical stability, computation efficiency, and implementation complexity

\subsection{ENERGY CONTROL CENTER}

Fig.1.1 is a schematic diagram showing the information flow between the various functions to be performed in an operation control centre computer system. The system gets its information about the power system from remote terminal units (RTU) that encode measurement transducer output and opened/closed status information into digital signals that are transmitted to the operations centre over communication circuits.

Network topology program:

Topological observability analysis is necessary to examine whether the relationship between measurement allocation and power system configuration is appropriate. In order to run the state estimator, we must know how 
the transmission lines are connected to the load and generator buses, this information is called network topology. Since the breakers and switches in any substation can cause the network topology to change, a program must be provided that reads the telemeter breaker/switch status indications and restructures the electrical model of the system Every time when a system configuration is changed for some reasons, a topological observability test should be executed prior to performing the state estimation to check one-to-one correspondence between measurements and buses. If this is not the case, observability analysis methods can provide the minimum set of additional measurements needed to restore observability.

State Estimation:

As seen in the Fig.1.1, the electric model of the power system transmission system is sent to the state estimator program together with the analog measurements. The output of the state estimator consists of all bus voltage magnitudes and phase angles, transmission line MW and MVAR flows calculated from the bus voltage magnitude and phase angles, and bus loads and generations calculated from the line flows. In real time environment the state estimator consists of different modules such as network topology processing, observability analysis, state estimation and bad data processing. Thus the state estimation program forms the heart of entire real time control of any power system.

The output of the state estimator i.e. $|\mathrm{V}|, \delta$, Pij, Qij together with latest model developed by network topology program the form the basis for the Economic Load Dispatch or minimum Emission dispatch, Reactive Power Dispatch, contingency analysis program etc.

In addition, the control centre can transmit control information such as raise/lower commands to generators and open/close commands to circuit breakers and switches. The analog measurements of generator output must be used directly by the AGC program whereas all the other data will be processed by the state estimator before being used by other programs

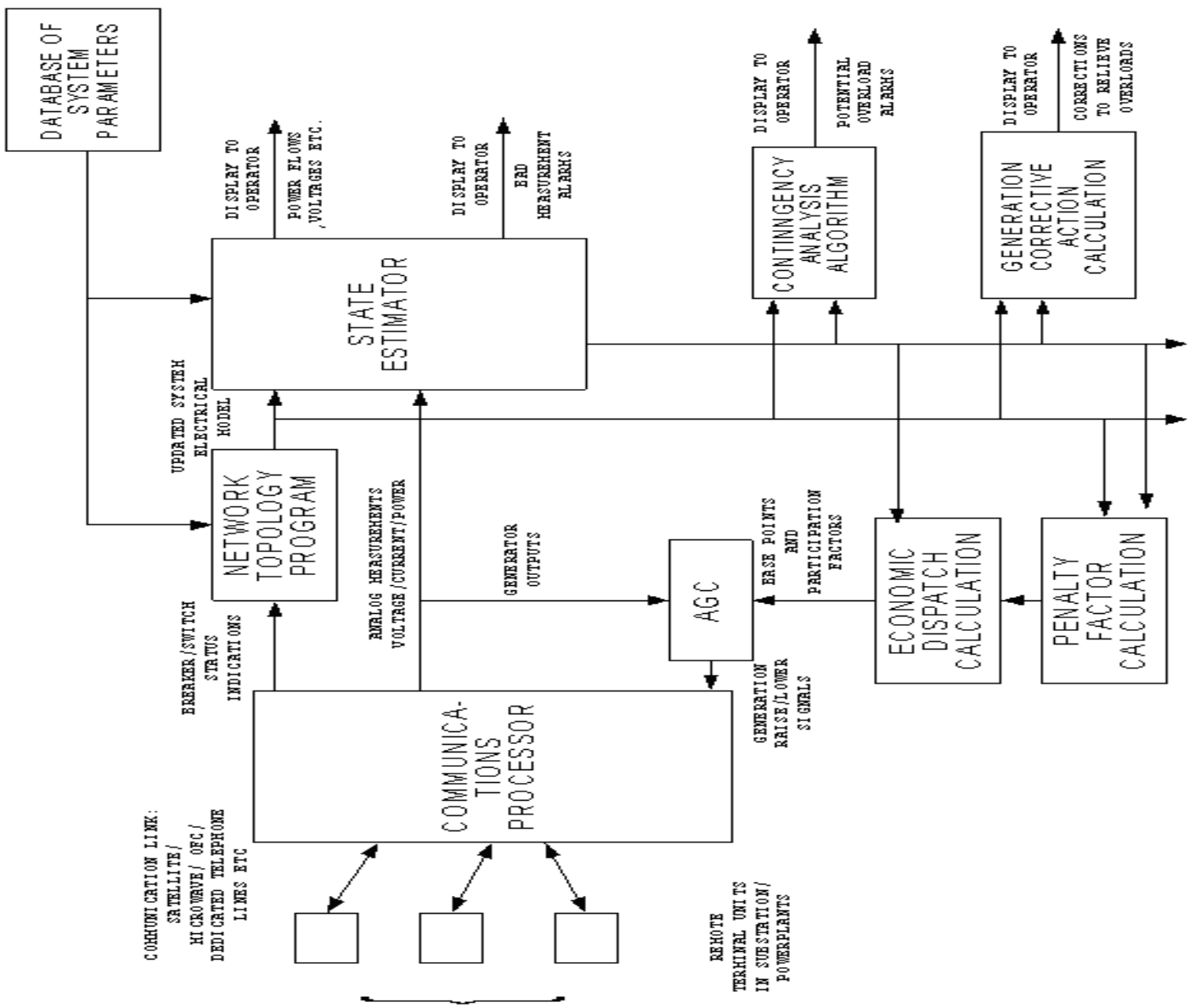

Fig 1.1 Energy control center system security schematic

\section{STATIC STATE ESTIMATION OF POWER SYSTEMS}

The goal of control center design is security control under the three states of power system operation: the normal, emergency, and restorative states. There are several functions performed by a control center in the classical energy 
management system (EMS) environment, and the most difficult of these to implement are those that run in a realtime environment. The key functions include state estimation, security monitoring, on-line load flow, security analysis, supervisory control, automatic generation control, automatic voltage/VAR control, and economic dispatch control [15]. These functions often interact in a complex manner, but all are aimed at providing the system operator with a coherent view of the system and/or carrying out the operator's decisions. Since all of these functions are directly dependent on state estimation, it is essential that the system operator trust the result.

In 1969 Fred Schweppe introduced the idea of using the redundant number of measurements, made available by the supervisory control and data acquisition (SCADA) system to statistically determine the state of the network. His proposition, the state estimator [14], was eventually accepted and serves as a basis for static state estimation.

Though power systems are dynamic, real-time systems, dynamic state estimation is generally not employed, reasons being- static state estimation presently fills the control center needs, and there are many difficulties in determining the dynamic system model and dynamic state estimation is computationally intensive[16]. Of these, the difficulty of defining a tractable, reliable model of the dynamic power system is the biggest inhibitor, due to the highly unpredictable and nonlinear nature of power systems.

The state estimator plays the essential role of a purifier, creating a complete and reliable database for security monitoring, security analysis and the various controls of a power system. The state estimator thus employs statistical methods to act as a tunable filter between the field data measurements and security and control functions.

The fundamental equation for the problem of power system state estimation (SE) can be formulated as

$$
z=h(x)+e
$$

Where $\mathrm{z}$ represents all measurements, including power injection, power flow and bus voltage magnitude measurements, $\mathrm{e}$ is the measurement noise vector, $\mathrm{x}$ is the state vector composed of the phase angles and magnitudes of the voltages at network buses, and $h($.$) stands for the nonlinear measurement functions in terms$ of state variables. It is always assumed that the parameters and observability of the systems are already determined in advance. It is to be noted that the measurements are never simultaneous, they are sequential, however at a very close interval and therefore the static state estimator assumes it to be snap-shot measurement [13], i.e. all measurements are assumed to be taken simultaneously.

\section{MODIFIED HOPFIELD NEURAL NETWORK METHOD FOR EQUALITY CONSTRAINED STATE ESTIMATION}

State Estimation processes a set of measurements to obtain the best estimate of the current state of the power system. The set of measurements includes telemetered measurements and pseudo-measurements. Telemetered measurements are the online telemetered data of bus voltages, line flows, injections, etc. Pseudo-measurements are manufactured data such as guessed MW generation or substation load demand based on historical data, in most cases. Telemetered measurements are subject to noise or error in metering, communication system, etc. The errors of some of the pseudo-measurements, especially the guessed ones, may be large. However, there is a special type of pseudo-measurements, known as the zero injections, for which the information contains no error. Zero injection occurs at a node, for example, representing a switching station where the power injection is equal to zero. Zero injection is an inherent property of such a node and no meter need to be installed but the information is always available. A state estimation algorithm must compute estimates, which satisfy exactly such constraints, independent of the quality of online measurements. The enforcing of constraints is in particular useful in networks, consisting of large unobservable parts of network or having very low measurement redundancy.

In its conventional form, the Weighted Least Square method does not enforce the equality and limit constraints explicitly. However, the constraints contain reliable information about physical restrictions and equipment limits and can be used to increase the quality of state estimation result. The zero injections can be represented by a set of equalities. Various methods have been proposed to process constraints, literature review section lists some of the proposed methods for solving Equality constrained State Estimation problem.

Various algorithms of State Estimation using the conventional computer are reaching a limit as far as the solution techniques are concerned, and as long as these computer based algorithms are used, faster methods can not be expected. However for security monitoring and control in power system, improvement in calculation time is always desired in order to obtain necessary information more quickly and accurately.

In recent years, it has been found that Artificial Neural Networks (ANN's) are well suited as computational tools for solving certain classes of complex problems, although software implementations of the algorithm on general-purpose computers can be too slow for time-critical applications, but the small number of computational 'primitives', suggests advantages of hosting ANN's on dedicated Neural Network Hardware (NNH) to maximize performance at a given cost target. ANN computations may be carried out in parallel, and special hardware devices are being designed and manufactured which take advantage of this capability.

In this chapter a new method for enforcing equality and limit constraints in State Estimation algorithm using a modified Hopfield neural network is presented. This method is tested for 6 bus system and IEEE 14 bus system. 
The main advantages of using the modified Hopfield neural network proposed in this work are i-) the internal parameters of the network are explicitly obtained by the valid-subspace technique of solutions, ii-) lack of need for adjustment of penalty factors for initialization of constraints, and iii-) for real time application, the modified Hopfield network offers simplicity of implementation in analog hardware or a neural network processor.(iv) training and testing of the neural network under human supervision is not required.

\section{STATE ESTIMATION WITH CONSTRAINTS}

State vector of an electric network consists of the complex voltages at the buses. Unmeasured tap positions of transformers may also be included into the state vector. A measurement vector consists of power flows, power injections, voltage and current magnitudes and tap positions of transformers. For a $\mathrm{N}$ bus system, the state vector $\mathrm{X}=[\delta, \mathrm{V}]^{\mathrm{T}}$, of dimension $\mathrm{n}=2 \mathrm{~N}-1$, consists of the $\mathrm{N}-1$ bus voltage angles $\delta_{\mathrm{i}}$ with respect to a reference bus and the $\mathrm{N}$ bus voltage magnitudes $\mathrm{V}_{\mathrm{i}}$ for $\mathrm{i}=1,2,3, \ldots . \mathrm{N}$.

The static state estimator measurement model is given as:

$$
\mathrm{z}=\mathrm{h}(\mathrm{X})+\mathrm{\epsilon}
$$

where $\mathrm{z}$ is the measurement vector, $\mathrm{h}($.$) is a vector of nonlinear functions, relating the measurement and state$ vectors, and $\epsilon$ is the vector of measurement errors.

The error-free data are modeled as equality constraints

$$
\mathrm{g}(\mathrm{X})=0
$$

Limits on some network variables are modeled as inequality constraints which can be expressed in a compact form by $\mathrm{p}$-dimensional functional inequalities

$$
\mathrm{f}(\mathrm{x}) \leq 0
$$

General nonlinear programming algorithms for the solution of a constrained minimization problem [24] are not efficient enough for the on-line application. Hence a neural network approach is used for solving this nonlinear programming problem.

Objective function

The objective is to minimize the weighted squared mismatch between measured and calculated quantities. Considering system to be observable and with $m>n$, where $m$ is the total number of measurements and $n$ is the number of state variables, the mathematical problem is given as follows:

$$
\mathrm{m} \text { in } \frac{1}{2}[\mathrm{Z}-\mathrm{h}(\mathrm{X})]^{\mathrm{T}} \mathrm{R}^{-1}[\mathrm{Z}-\mathrm{h}(\mathrm{X})]
$$

Subject to the equality and inequality constraints as defined below.

The diagonal matrix $\mathrm{R}^{-1}$ represents the weights of the individual measurements in the objective function.

\section{RESULTS}

The proposed method is tested on Ward Hale 6 bus system and modified IEEE 30 bus system and the results are compared with conventional and optimization techniques. Since neuron output consists of different terms, linear relationship between input and output doesn't holds well. Hence sigmoid function is considered in this case. Convergence criterion adopted is $€<0.0001$. Voltage limits for load buses are $V_{\min }=0.9, V_{\max }=1.1$ and for PV bus are $\mathrm{V}_{\min }=1, \mathrm{~V}_{\max }=1.06$. Tap limits are $\mathrm{T}_{\min }=0.9, \mathrm{~T}_{\max }=1.1$ and shunt limits taken are $\mathrm{S}_{\min }=0$ and $\mathrm{S}_{\max }=, \mathrm{A}=0.5$, $\mathrm{B}=0.04$ (It is to be noted that convergence is independent of values of $\mathrm{A}, \mathrm{B}$ ).

The data for Ward Hale 6 bus system is given at Appendix B.

The results are shown in table 7.1. Execution time on typical PC is $0.516 \mathrm{sec}$.

Table 4.1 Ward Hale 6 bus system

\begin{tabular}{|c|c|c|c|c|c|}
\hline Variables & Non fuzzy[42] & Fuzzy[42] & GA[43] & PSO[43] & HHN \\
\hline V1 & 1.09 & 1.1 & 1.0225 & 1.023 & 1.1 \\
\hline V2 & 1.15 & 1.15 & 1.1 & 1.1 & 1.1 \\
\hline V3 & 1.0 & 1.01 & 0.99 & 1.0 & 1.049713 \\
\hline V4 & 1.0 & 1.01 & 0.917 & 0.918 & 1.049631 \\
\hline V5 & 1.00 & 1.01 & 0.969 & 0.9696 & 0.975035 \\
\hline V6 & 0.98 & 0.994 & 0.9 & 0.9019 & 0.975082 \\
\hline Q1 & 0.363 & 0.353 & 0.423 & 0.927 & 0.119356 \\
\hline Q2 & 0.193 & 0.194 & 0.378 & 0.579 & 0.143057 \\
\hline Q4 & 0.05 & 0.05 & 0.05 & 0.05 & 0.055 \\
\hline Q6 & 0.055 & 0.055 & 0.055 & 0.055 & 0.05 \\
\hline T65 & 0.96 & 0.98 & 0.9 & 0.9 & 0.9725 \\
\hline T43 & 0.98 & 0.99 & 0.9 & 0.9 & 0.9090 \\
\hline Loss & $\mathbf{8 . 9 3}$ & $\mathbf{8 . 7 7}$ & $\mathbf{8 . 1 7 4 6}$ & $\mathbf{8 . 1 7 4 5}$ & $\mathbf{8 . 1 5 6 7 7 2}$ \\
\hline
\end{tabular}




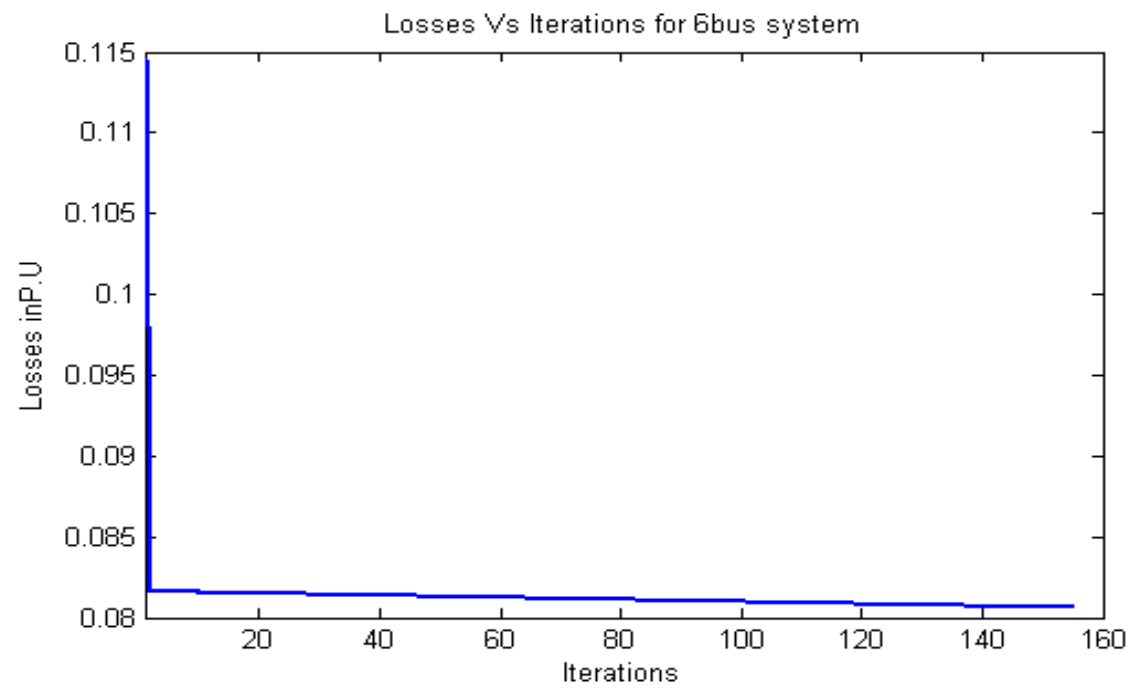

Fig4.2

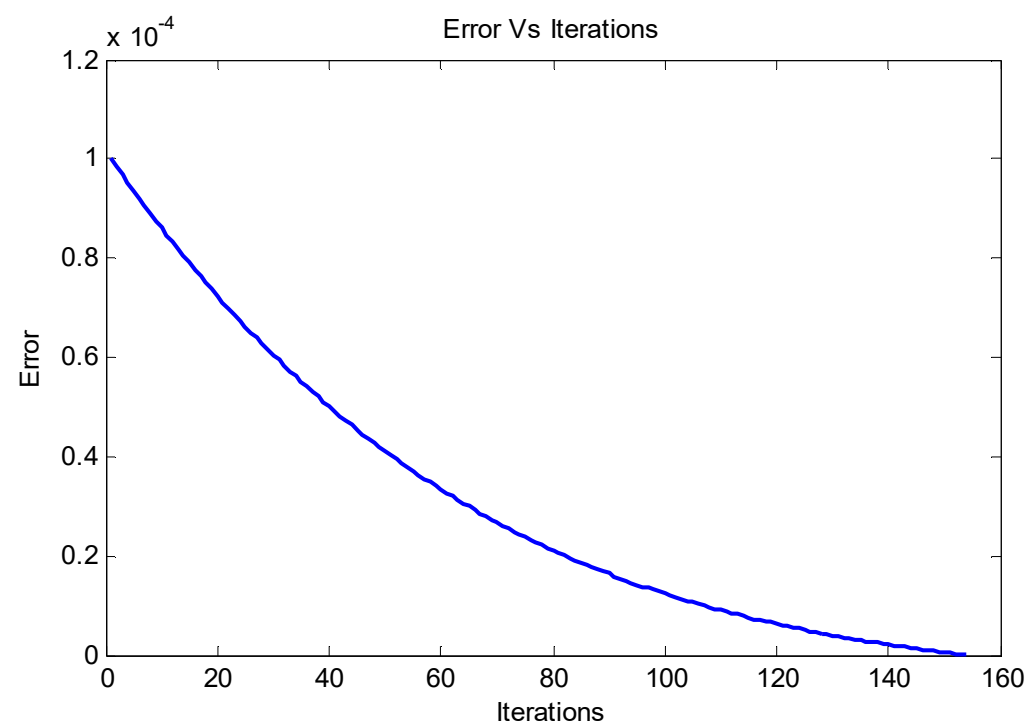

Fig 4.3

\subsection{Modified IEEE 30 bus system}

The data for Modified IEEE 30 bus system is given at Appendix B. The results are shown in Table 7.2. Execution time on a typical PC is found to be is $2.187 \mathrm{sec}$ 
Table 4.2 Modified IEEE 30 bus system

\begin{tabular}{|c|c|c|c|c|}
\hline Variables & GA[43] & Classical PSO [43] & Improved PSO [43] & HHN \\
\hline $\mathrm{V}_{1}$ & 1.0 & 1.018 & 1.015 & 1.031082 \\
\hline $\mathrm{V}_{2}$ & 0.999 & 1.012 & 1.0048 & 1.0338 \\
\hline $\mathrm{V}_{5}$ & 0.974 & 1.013 & 1.0017 & 1.022362 \\
\hline $\mathrm{V}_{8}$ & 1.007 & 0.994 & 0.99 & 1.026687 \\
\hline $\mathrm{V}_{11}$ & 1.0894 & 1.04 & 1.0019 & 1.0913 \\
\hline $\mathrm{V}_{13}$ & 1.088 & 1.016 & 0.993 & 1.025466 \\
\hline $\mathrm{Q}_{1}$ & 0.597 & 0.147 & 0.433 & 0.6233 \\
\hline $\mathrm{Q}_{2}$ & 0.755 & 0.348 & 0.452 & -0.041101 \\
\hline $\mathrm{Q}_{5}$ & -0.128 & 0.0532 & 0.0565 & 0.0621 \\
\hline $\mathrm{Q}_{8}$ & 0.052 & 0.144 & 0.156 & 0.15 \\
\hline $\mathrm{Q}_{11}$ & 0.1725 & 0.166 & 0.068 & 0.15 \\
\hline $\mathrm{Q}_{13}$ & 0.0386 & 0.0306 & 0.0636 & 0.070258 \\
\hline $\mathrm{Q}_{17}$ & 0.045 & 0.025 & 0.05 & 0.031262 \\
\hline $\mathrm{Q}_{18}$ & 0.045 & 0.05 & 0.055 & 0.049293 \\
\hline $\mathrm{Q}_{23}$ & 0.045 & 0.055 & 0.055 & 0.055 \\
\hline $\mathrm{Q}_{27}$ & -0.01 & 0.02 & 0.03 & 0.01 \\
\hline $\mathrm{T}_{1,3}$ & 0.975 & 1.05 & 1.025 & 1.0625 \\
\hline $\mathrm{T}_{2,4}$ & 1.0 & 1.025 & 1.0125 & 1.0875 \\
\hline $\mathrm{T}_{5,7}$ & 0.9625 & 1.0125 & 1.025 & 0.925 \\
\hline $\mathrm{T}_{9,10}$ & 1.0 & 1.0 & 0.9875 & 1.0625 \\
\hline $\mathrm{T}_{12,13}$ & 0.9875 & 1.0625 & 1.0625 & 1.0875 \\
\hline $\mathrm{T}_{18,19}$ & 1.0375 & 1.075 & 1.0375 & 0.9375 \\
\hline $\mathrm{T}_{10,17}$ & 0.95 & 0.95 & 0.95 & 0.925 \\
\hline $\mathrm{T}_{23,24}$ & 1.0625 & 1.05 & 1.0375 & 1.05 \\
\hline $\mathrm{T}_{27,29}$ & 1.0125 & 1.0 & 1.0 & 1.05 \\
\hline $\mathrm{T}_{8,28}$ & 1.0 & 1.0 & 1.0 & 1.025 \\
\hline Loss & 4.2716 & 4.1501 & 4.1396 & 3.94198 \\
\hline
\end{tabular}

Losses Vs Iterations for IEEE 30 bus system

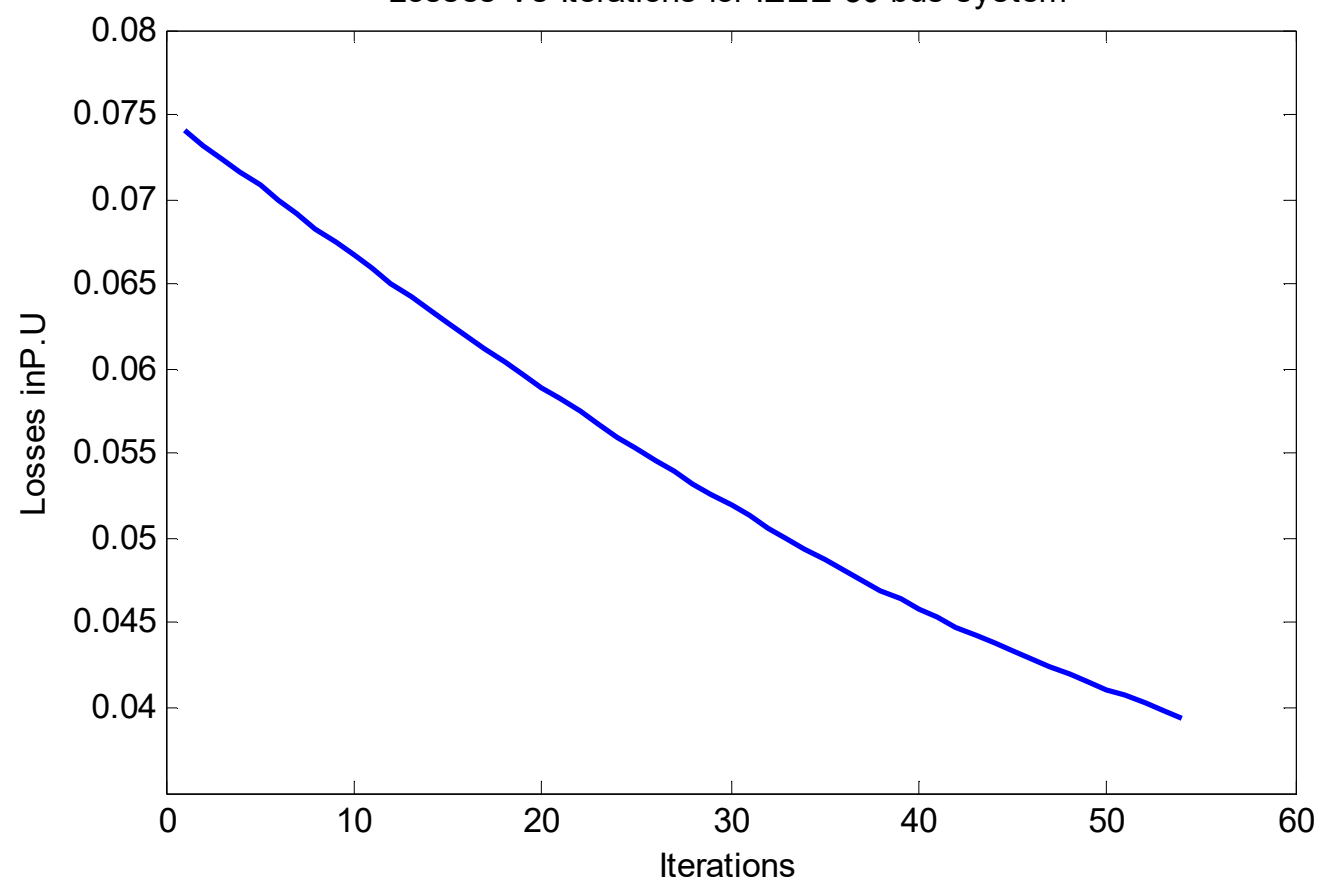

Fig 4.3 Losses Vs Iterations for IEEE 30 bus system 


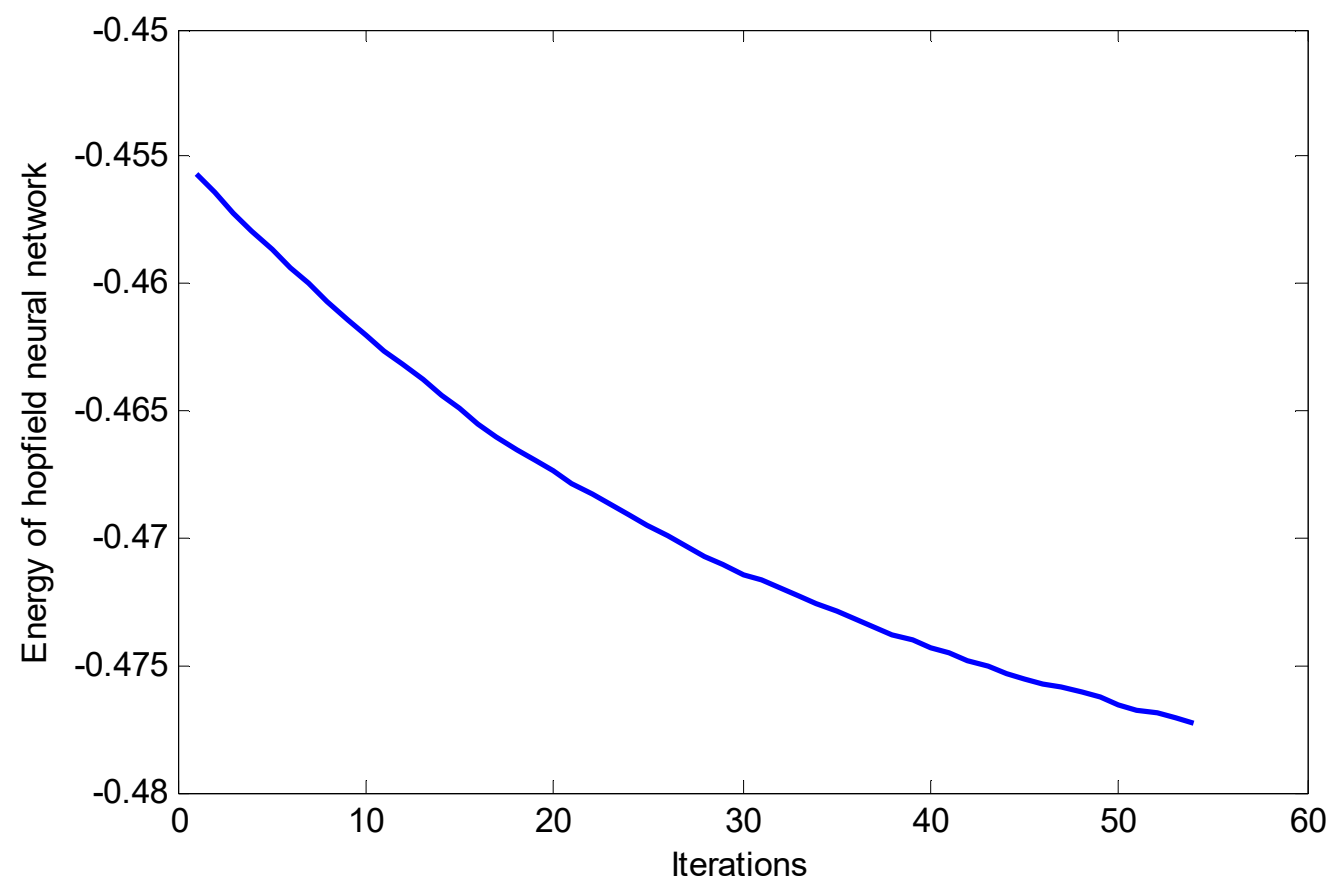

Fig 4.4 Energy Vs Iterations for IEEE 30 bus system

\section{CONCLUSIONS}

Releasing the importance of neural networks for hardware implementation, in this project neural network based solutions were developed to solve most important computational tasks in real time control of power systems. A Hopfield neural network model was developed for determination of topological observability of power system. This neural network has been tested on test systems of 5 bus 10 measurement system and 9 bus 14 measurement system. The results obtained are crosschecked with those of conventional root base observability determination. It is found that neural network is far simpler in coding compared to conventional method which involves lot many complexities.

The software package has been developed (in MATLAB) for reactive power optimization using Hopfield neural network. The so developed module is tested on Ward Hale 6 bus system and IEEE 30 bus system. A comparison is made between fuzzy, non fuzzy, classical P.S.O and improved P.S.O. It is found that the ANN approaches provides efficient and comparable results to those of conventional and optimization methods.

Finally the papaer work has provided the opportunity to gain good experience in using ANN, PSO and other conventional techniques to power system related topics like state estimation, Observability, active power optimization, reactive power dispatch etc.

\section{References}

[1] Wu F.F., Liu W.H.E., Holtan L., Gjelsvik A., Aam S., "Comparison of Different Methods for State Estimation," IEEE Transaction On Power Systems, Vol. 3, No. 4, November 1988, pp 1798-1806.

[2] F.C. Schweppe, J. Wildes, D.B. Rom, "Power System Static-State Estimation, Parts I, II and III", IEEE Transactions on Power Apparatus and Systems, Vol. PAS-89, No. 1, January 1970, pp. 120-135.

[3] A. Garcia, A. Monticelli, and P. Abreu, "Fast Decoupled State Estimation and Bad Data Processing," IEEE Transaction Power Apparatus and systems. Vol. PAS-98, pp. 1645-1652, Oct. 1979.

[4] A. Simoes Costa, and V. H. Quintana, "A Robust Numerical Technique for Power System State Estimation," IEEE Transaction Power Apparatus and systems. Vol. PAS-100, pp. 691-698, Feb. 1981.

[5] F. C. Aschmoneit, N. M. Peterson, and E. C. Adrian, "An Orthogonal Row Processing Algorithm for Power System Sequential State Estimation,” IEEE Transaction Power Apparatus and systems. Vol. PAS-100, pp. 3791-3800, Aug. 1981.

[6] J. W. Wang and V. H. Quintana, "A Decoupled Orthogonal Row Processing Algorithm for Power System State Estimation,” IEEE Transaction Power Apparatus and systems. Vol. PAS-103, pp. 2337-2344, Aug. 1984.

[7] A. Monticelli, C. A. F. Murati, and F. F. Wu, "A Hybrid State Estimator: Solving Normal Equations by Orthogonal Transformations," IEEE Transaction Power Apparatus and systems. Vol. PAS-105, pp. 3460- 
3468, Dec. 1985.

[8] F. C. Aschmoneit, N. M. Peterson, and E. C. Adrian, "State Estimation with Equality Constraints," in Proc. 10th PICA Conf., Toronto, Canada, May 1977, pp. 427-430.

[9] A. Simoes-Costa, S. Seleme, and R. Salgado, "Equality Constraints in Power System State Estimation via Orthogonal Row-Processing Techniques," in Proc. IFAC Conf. Electrical Energy Systems., Rio de Janeiro, Brazil, 1985, pp. 43-49.

[10] A. Gjelsvik, S. Aam, and L. Holten, “Hachtel's Augmented Matrix method-A rapid method for Improving Numerical Stability in Power System Static State Estimation," IEEE Transaction Power Apparatus and systems. Vol. PAS-104, pp. 2987-2993, Nov. 1985.

[11] W. E. Liu, F. F. Wu, L. Holten, A. Gjelsvik, and S. Aam, "Computational issues in the Hachtel's Augmented Matrix method for Power System State Estimation,” in Proc. Power System Computation Conf., Lisbon, Portugal, 1987.

[12] F. Alvarado and W. Tinney, "State Estimation Using Augmented Blocked Matrices," IEEE Transaction Power Systems, vol. 5, pp. 911-921, Aug. 1990.

[13] R. R. Nucera and M. L. Gilles, "A Blocked Sparse Matrix Formulation for the solution of EqualityConstrained State Estimation,” IEEE Transaction Power Syst., vol. 6, pp. 214-224, Feb. 1991.

[14] E. Kliokys and N. Singh, "Minimum Correction Method for Enforcing limits and Equality Constraints in State Estimation Based on Orthogonal Transformations," IEEE Transaction Power Systems., vol. 15, pp. 1281-1286, Nov. 2000.

[15] A. Clements and B.F. Wollenberg. "An Algorithm for Observability Determination in Power System State Estimation,'’ IEEE PES Summer Meeting, Paper A 75 447-3, San Francisco, July 1975.

[16] V.H. Quintana. A. Shoes-Costa, and A. Mandcl, "Power System Observability Using a Direct GraphTheoretic Approach," IEEE Transaction Power App. and Systems, Vol. 101. No. 3, pp. 617-626, March 1982.

[17] Monticelli and F.F. Wu, "Network Observability: Identification of Observable Islands and Measurement Placement," IEEE Transaction on Power Apparatus and Systems., Vol. Pas-104, No. 5, pp. 1035- 1041, May 1985.

[18] A.Bargiela, et al., “Observability Determination in Power System State Estimation,” IEEE Transaction On Power Syst., Vol. PWRS-1, No. 2, p. 108-114, May 1986. 